\title{
A Sequel to the Eteplirsen Saga: Eteplirsen Is Approved in the United States but Was Not Approved in Europe
}

\author{
Annemieke Aartsma-Rus ${ }^{1}$ and Nathalie Goemans ${ }^{2}$
}

Eteplirsen was approved for the treatment of eligible patients with Duchenne muscular dystrophy (DMD) in September 2016 in one of the most, if not the most, controversial approvals ever made by the Food and Drug Administration of the United States. Two years later, the Committee for Human Medicinal Products (CHMP) of the European Medicines Agency gave a negative opinion for eteplirsen treatment. They had done so as well in May 2018, after which Sarepta (the company developing eteplirsen) appealed and a new evaluation was initiated, including a Scientific Advisory Group (SAG) meeting involving DMD experts and patient representatives. However, after reevaluation the opinion of the CHMP remained negative. In this commentary, we outline how differences in the perspective of FDA and EMA can lead to a DMD therapy being approved by FDA but not EMA, and vice versa.

Keywords: Duchenne muscular dystrophy, regulatory approval, clinical trials

$\mathbf{E}$ TEPLIRSEN WAS APPROVED for the treatment of eligible patients with Duchenne muscular dystrophy (DMD) in September 2016 in one of the most, if not the most, controversial approvals ever made by the Food and Drug Administration (FDA) of the United States [1]. Two years later, the Committee for Human Medicinal Products (CHMP) of the European Medicines Agency (EMA) gave a negative opinion for eteplirsen treatment (http://investorrelations.sarepta.com/ news-releases/news-release-details/sarepta-receives-negativechmp-re-examination-opinion-eteplirsen and https://www.ema .europa.eu/news/meeting-highlights-committee-medicinalproducts-human-use-chmp-17-20-september-2018).

They had done so as well in May 2018, after which Sarepta (the company developing eteplirsen) appealed and a new evaluation was initiated, including a Scientific Advisory Group (SAG) meeting involving DMD experts and patient representatives. However, after reevaluation the opinion of the CHMP remained negative. In this commentary, we outline how differences in the perspective of FDA and EMA can lead to a DMD therapy being approved by FDA but not EMA, and vice versa.

DMD is a severely progressive disease caused by the lack of functional dystrophin protein due to mutations in the $D M D$ gene. The disease is rare, occurring in 1 in 5,000 newborn boys and characterized by the irreversible loss of muscle tissue and function, leading to premature death in the secondfourth decade of life. Eteplirsen is a phosphorodiamidate oligonucleotide that aims to restore dystrophin production by modulating the splicing of the mutated dystrophin transcript.
It works in a mutation-specific manner, and as such, eteplirsen would only apply to $13 \%-14 \%$ of all patients $[2,3]$. For a detailed time line of eteplirsen development, we refer the reader to a previous "Issues in Development" article [1]. In brief, the accelerated approval was based on minimal increases in dystrophin expression in biopsies of 11 patients after 180 weeks of treatment $(0.93 \%$ increase $)$ and 13 patients after 48 weeks of treatment $(0.28 \%$ increase $)$. As such, the drug was approved based on a surrogate endpoint (dystrophin increase), which was considered reasonably likely to predict future clinical benefit and a good safety profile.

Limited functional data are available as of this moment. Twelve patients have been treated weekly with intravenous infusions of eteplirsen for over 3 years and have been followed by regular 6-min walk tests (6MWT). Given that this extension study has no control group, the 6-min walk distance (6MWD) could only be compared with the available 3-year natural history data from age- and stage-matched patients with similar characteristics from Belgium and Italy [4]. This comparison suggests a slower disease progression for the eteplirsen treatment. However, the small sample size and known heterogeneity in disease course [5], as well as the applicability of contemporaneous natural history data as comparison, even if collected in same circumstances of standards of care, were questioned. As such, FDA was not convinced yet about functional benefit and requested that Sarepta provide data on functional benefit from eteplirsen treatment by 2021 as a condition of the accelerated approval [1].

Now why would regulators in Europe presented with the same data come to a different conclusion? First, it is

\footnotetext{
${ }^{1}$ Department of Human Genetics, Leiden University Medical Center, Leiden, the Netherlands.

${ }^{2}$ Department of Child Neurology, University Hospitals Leuven, Leuven, Belgium.
} 
important to understand that the position of using a surrogate endpoint as a primary endpoint differs between FDA and EMA. FDA based its decision on the surrogate (increased dystrophin expression) on the assumption that there is a reasonable probability of a functional benefit in the future. With EMA, a surrogate endpoint first needs to be qualified, that is, one first has to provide functional data to confirm the correlation between the surrogate endpoint and clinical benefit [6]. Or in this case show that very minor increases in dystrophin indeed result in functional effects. From stakeholder discussions between patients, regulators from Europe, and academics, it became apparent that dystrophin increases can be used as a pharmacodynamic marker, to confirm mechanism of action of a drug, but without prior validation, they are not acceptable as a primary endpoint [6].

In light of this, it does not come as a surprise that faced with the same data, the CHMP gave a negative opinion. Actually, in a strange reversal of fortunes, another drug that aims at dystrophin restoration, ataluren, developed by PTC Therapeutics, received conditional approval from the EMA [7], while it was not approved by the FDA (http://ir.ptcbio .com/news-releases/news-release-details/ptc-therapeuticsreceives-formal-dispute-resolution-request). Ataluren is also a mutation-specific therapy and applies only to DMD patients who have a nonsense mutation ( $\sim 10 \%-13 \%$ of patients).

Here, the company conducted a double-blinded, placebocontrolled trial in 174 patients [8]. They did not present data on dystrophin levels. However, 6MWD results were available from a placebo-controlled trial. At first, the primary endpoint (30-m differences) was not met. However, after removing data of two patients who suffered a bad fall before the 6MWT during post hoc analysis, patients treated with the appropriate dose on average walked $31.4 \mathrm{~m}$ further than the placebo group after 48 weeks of treatment. Furthermore, in a specific subgroup of patients, that is, those most likely to show a response in the 6MWD during 48 weeks, a difference of $>50 \mathrm{~m}$ was observed.

This was deemed clinically beneficial and the drug was approved conditionally for ambulant DMD patients with a nonsense mutation aged 5 and older [7]. Additional information to confirm the functional efficacy of the drug had to be provided by PTC. Unfortunately, no significant difference was observed between placebo and ataluren-treated patients in this confirmatory trial [9]. However, again a difference of $>50 \mathrm{~m}$ in 6MWD was observed in the prespecified subgroup most likely to show a treatment effect.

In light of this, the CHMP prolonged the conditional approval, requesting a second confirmatory trial to show drug efficacy by September 2021 [10] to convert the conditional approval into a full approval, or failing that, revoking of the conditional approval. PTC filed a new drug application (NDA) to FDA based on the combined data of the two placebo-controlled trials [8,9]. However, FDA refused to file the NDA, since significant differences were only observed after unblinding and post hoc analyses. PTC appealed, and FDA indicated, after an advisory committee, that is unable to approve ataluren based on the currently available data [11].

These examples outline how the different stances can result in drugs being approved by one regulatory agency and not by another. However, while the CHMP gave a negative opinion for eteplirsen, Sarepta indicated that it did appreciate the willingness of EMA to consider different approaches to provide additional data required for approval for this (and therefore also future) splice modulating compound(s). Given that eteplirsen treatment applies to the largest subgroup of patients, while other splice modulating compounds apply to increasingly smaller groups of patients, this is good news.

When a drug is applicable to only $2 \%$ of patients with a rare disease, the number of patients simply is not sufficient to conduct placebo-controlled trials. In light of this, the collaborative Trajectory Analysis Program (cTAP) compared 6MWD trajectories of patients from natural history cohorts from 40 centers in Europe and United States with trajectories of 375 patients receiving placebo treatment from 5 randomized, double-blinded clinical trials. Interestingly, the trajectories did not differ significantly, suggesting that natural history may in the future be used as a comparator rather than a placebo group (Goemans et al., unpublished results).

\section{Author Disclosure Statement}

A.A.R. discloses being employed by LUMC, which has patents on exon skipping technology. As coinventor of some of these patents A.A.R. is entitled to a share of royalties. A.A.R. further discloses being or having been an ad hoc consultant for PTC Therapeutics, BioMarin Pharmaceuticals, Inc., Sarepta Therapeutics, Alpha Anomeric, Eisai, Vertex, Global Guidepoint GLG consultancy, Deerfield, Grünenthal, and Bioclinica, and being a member of the scientific advisory boards of ProQR and Philae Pharmaceuticals. Remuneration for these activities is paid to LUMC. LUMC also received speaker honoraria from PTC Therapeutics and BioMarin Pharmaceuticals. N.G. has been a principal investigator for trials sponsored by PTC Therapeutics, GlaxoSmithKline, Prosensa, BioMarin Pharmaceuticals, and Lilly; has received speaker honoraria from PTC Therapeutics, Biogen, and BioMarin; and has been on the advisory board for PTC Therapeutics, Italfarmaco, Summit Therapeutics, and Roche.

\section{References}

1. Aartsma-Rus A and AM Krieg. (2017). FDA approves eteplirsen for duchenne muscular dystrophy: the next chapter in the eteplirsen saga. Nucleic Acid Ther 27:1-3.

2. Aartsma-Rus A, I Fokkema, J Verschuuren, I Ginjaar, J van Deutekom, GJ van Ommen and JT den Dunnen. (2009). Theoretic applicability of antisense-mediated exon skipping for Duchenne muscular dystrophy mutations. Hum Mutat 30:293-299.

3. Bladen CL, D Salgado, S Monges, ME Foncuberta, K Kekou, K Kosma, H Dawkins, L Lamont, AJ Roy, et al. (2015). The TREAT-NMD DMD Global Database: analysis of more than 7,000 Duchenne muscular dystrophy mutations. Hum Mutat 36:395-402.

4. Mendell JR, N Goemans, LP Lowes, LN Alfano, K Berry, J Shao, EM Kaye, E Mercuri and Eteplirsen Study Group and Telethon Foundation DMD Italian Network. (2016). Longitudinal effect of eteplirsen versus historical control on ambulation in Duchenne muscular dystrophy. Ann Neurol 79:257-271.

5. Goemans $\mathrm{N}, \mathrm{M}$ van den Hauwe, R Wilson, A van Impe, $\mathrm{K}$ Klingels and G Buyse. (2013). Ambulatory capacity and disease progression as measured by the 6-minute-walkdistance in Duchenne muscular dystrophy subjects on daily corticosteroids. Neuromuscul Disord 23:618-623.

6. Aartsma-Rus A, V Straub, R Hemmings, M Haas, G Schlosser-Weber, V Stoyanova-Beninska, E Mercuri, F Muntoni, B Sepodes, E Vroom and P Balabanov. (2017). 
Development of exon skipping therapies for Duchenne muscular dystrophy: a critical review and a perspective on the outstanding development issues. Nucleic Acid Ther 27:251-259.

7. Haas M, V Vlcek, P Balabanov, T Salmonson, S Bakchine, G Markey, M Weise, G Schlosser-Weber, H Brohmann, et al. (2015). European Medicines Agency review of ataluren for the treatment of ambulant patients aged 5 years and older with Duchenne muscular dystrophy resulting from a nonsense mutation in the dystrophin gene. Neuromuscul Disord 25:5-13.

8. Bushby K, R Finkel, B Wong, R Barohn, C Campbell, GP Comi, AM Connolly, JW Day, KM Flanigan, et al. (2014). Ataluren treatment of patients with nonsense mutation dystrophinopathy. Muscle Nerve 50:477-487.

9. McDonald CM, C Campbell, RE Torricelli, RS Finkel, KM Flanigan, N Goemans, P Heydemann, A Kaminska, J Kirschner, et al. (2017). Ataluren in patients with nonsense mutation Duchenne muscular dystrophy (ACT DMD): a multicentre, randomised, double-blind, placebo-controlled, phase 3 trial. Lancet 390:1489-1498.
10. European Public Assessment Report (EPAR) for translarna. https://www.ema.europa.eu/documents/product-information/ translarna-epar-product-information_en.pdf

11. Response letter from FDA to PTC about translarna filing: http://ir.ptcbio.com/news-releases/news-release-details/ptctherapeutics-receives-complete-response-letter-atalurens-nda

Address correspondence to: Annemieke Aartsma-Rus, PhD Department of Human Genetics Leiden University Medical Center Albinusdreef 2 2333 ZA Leiden The Netherlands

E-mail: a.m.rus@lumc.nl

Received for publication October 9, 2018; accepted after revision November 10, 2018. 